\title{
Influence of Microstructure on the Rheological Behavior of Dense Particle Gels
}

\author{
Hans M. Wyss, Aylin M. Deliormanli, Elena Tervoort, and Ludwig J. Gauckler \\ ETH Zurich, Nonmetallic Materials, Dept. of Materials, Zurich CH-8092, Switzerland \\ DOI 10.1002/aic.10296 \\ Published online in Wiley InterScience (www.interscience.wiley.com).
}

\begin{abstract}
Rheological measurements are performed on highly concentrated alumina gels. By using an in situ mechanism based on enzyme-catalyzed internal reactions, we are able to form gels of highly concentrated particles without disturbing the microstructures that develop during the gelation process. These gels are produced by two different destabilization mechanisms: Either the $\mathrm{pH}$ of the suspension is shifted toward the isoelectric point $(\Delta p H$ method) or the ionic strength of the suspension is increased at a constant $p H(\Delta I$ method). The two destabilization mechanisms lead to gels of significantly different microstructures. We find notable differences in the rheological behavior of the two systems, suggesting a bond-bending mechanism for stress transmission in the case of $\Delta p H$ gels and a bond-stretching mechanism in the case of $\Delta I$ gels. In addition, for both kinds of gels we compare the in situ properties to those obtained after altering the microstructure by shearing. Results suggest an increase in elastic and yield properties of concentrated particle gels with decreasing homogeneity of their microstructures. (๑) 2004 American Institute of Chemical Engineers AIChE J, 51: 134-141, 2005
\end{abstract}

\section{Introduction}

Controlling the rheological behavior of concentrated particle suspensions and gels is a key issue in the production of various products, such as food, paint, concrete, or ceramics. It is determined by the interparticle forces, the size and shape of the particles, the particle volume fraction, and the microstructure.

For particle gels formed at low volume fractions, the dependency of the rheology on these parameters is reasonably well established. The structure of these gels is accurately described as fractal (Weitz and Oliveria, 1984): the mass $m_{c}$ of aggregates scales with their size $R_{c}$ as a power law $m_{c} \propto R_{c}^{d_{f}}$, with $d_{f}<3$ the fractal dimension.

Accordingly, the density $\rho_{c}$ of the clusters scales as $\rho_{c} \propto$ $R_{c}^{d_{f}-3}$. When $\rho_{c}$ equals the volume fraction of the particles, an interconnected network of fractal clusters is formed. The size

Current address of A. M. Deliormanli: Izmir Institute of Technology, Chemical Engineering Dept., Gülbahce Köyü, Urla, Turkey.

Current address of H. M. Wyss: Harvard University, Physics \& DEAS, ESL 227, 40 Oxford Street, Cambridge, MA 02138.

Correspondence concerning this article should be addressed to L. J. Gauckler at ludwig.gauckler@mat.ethz.ch.

(C) 2004 American Institute of Chemical Engineers
$R_{c}$ of the clusters in a gel thus scales with $\phi$ as a power law $R_{c}$ $\propto \phi^{1 /\left(d_{f}-3\right)}$. Based on a theoretical description of the sizedependent elasticity of a single fractal cluster (Kantor and Webman, 1984), one can show that the rheological properties of a fractal gel network will then also scale with the particle volume fraction (Brown and Ball, 1985; Shih et al., 1990; Wu and Morbidelli, 2001). This theoretical description is in good agreement with various experiments and computer simulations, and provides a well-defined relation between microstructure and mechanical behavior of diluted gel networks.

For highly concentrated gels, however, much less is known on the influence of microstructure on the mechanical behavior. This is partly attributed to the considerable difficulties involved in experimentally characterizing microstructures of highly concentrated systems. More important, however, is the fact that conventional destabilization techniques do not allow the controlled formation of particle gels with significantly different microstructures at high volume fractions.

In a standard rheological experiment performed on a particle gel, the formation of the gel is achieved by directly adding salt or acid/base to the liquid phase of a stable suspension. The mechanical properties of both stable particle suspensions (Fa- 
gan and Zukoski, 1997) and gel networks (Channell and Zukoski, 1997; Channell et al., 2000; Rueb and Zukoski, 1997) have been extensively studied in recent years. However, for highly concentrated suspensions it is generally not possible to achieve an undisturbed gelation by the conventional addition of salt or acid/base because of the elevated aggregation rates in these systems. Strong aggregation already takes place before the salt or acid/base can be homogeneously distributed by diffusion or mixing. The microstructure of the resulting particle gels is therefore highly inhomogeneous and irreproducible. In rheological measurements, reproducible results are usually not achieved unless the samples are strongly sheared before conducting the measurements. It is reasonable to assume that the microstructure of the resulting gels will in this case be governed to a large extent by the mixing process, and not by the gelation process itself.

Thus, in situ destabilization techniques that allow for the formation and characterization of undisturbed particle gels are required.

Direct coagulation casting (DCC; Gauckler et al., 1999a,b), which was developed for the processing of ceramics, offers the possibility of changing $\mathrm{pH}$ and/or ionic strength in a homogeneous and continuous way. Thus electrostatically stabilized suspensions can be destabilized in situ by either shifting the $\mathrm{pH}$ to the isoelectric point ( $\Delta \mathrm{pH}$ method) or by increasing the ionic strength $I$ ( $\Delta I$ method) without disturbing the colloid history. This is achieved by the use of an enzyme-catalyzed internal chemical reaction, such as the hydrolysis of urea, catalyzed by the enzyme urease. The reaction is time delayed and the speed of the reaction is controlled by both enzyme concentration and temperature.

In this study, we use the DCC technique to achieve a true in situ destabilization of the suspensions, resulting in the undisturbed formation of particle gels, exhibiting reproducible microstructures. Reproducible results are obtained in rheological measurements on these systems, even when they are not subjected to shear before measurement.

Previous studies on highly concentrated wet green bodies formed by DCC show that the chemical pathway of destabilization (that is, $\Delta \mathrm{pH}$ or $\Delta I$ ) has a strong influence on both structure and mechanics of these systems. Greater than tenfold higher yield stress and elastic modulus values were found for $\Delta I$ - compared to $\Delta \mathrm{pH}$-destabilized systems in compression tests on highly concentrated particle gels (Balzer et al., 1999a). This finding is rather surprising, given that the interparticle forces in the final state are expected to be stronger for $\Delta \mathrm{pH}$ destabilized systems compared to $\Delta I$ systems (Chang et al., 1994).

In a parallel study, we have examined the microstructure of concentrated particle gels by using high-pressure freezing in combination with Cryo-SEM on model systems of monodispersed silica spheres (Wyss et al., 2002, 2004). We found an inhomogeneous microstructure for $\Delta I$-destabilized gels and a very homogeneous structure for $\Delta \mathrm{pH}$-destabilized gels. This result is confirmed by a recent light-scattering study, where the degree of inhomogeneity in concentrated alumina gels was estimated from changes in the static transmission during the destabilization process (Wyss et al., 2001). The observed differences in microstructure for the two different destabilization methods can be understood if we consider the different aggregation kinetics for the two cases. Although in the final stages of destabilization the interparticle potentials are governed by attractive van der Waals forces in both processes, the potentials are fundamentally different in the earlier stages of destabilization. Whereas in the $\Delta I$ method the Debye screening length gets shorter with time and the magnitude of the zeta potential remains essentially unchanged, for the $\Delta \mathrm{pH}$ method, the screening length remains essentially unchanged and the zeta potential decreases. This leads to a rapid change from repulsive to purely attractive for the $\Delta \mathrm{pH}$ destabilization, resulting in diffusion limited aggregation. On the contrary, for the $\Delta I$ method, a shallow secondary minimum occurs in the interaction potential, and the energy barrier to the primary minimum decreases more slowly. This situation allows for bonds to be broken and reformed in the secondary minimum. Irreversible aggregation occurs only at a later stage in the process, when the particles overcome the energy barrier to the primary minimum. With this in mind, it is reasonable to assume that $\Delta I$ destabilization would lead to locally more densely packed structures because particles are able to escape from their bonds and rearrange into energetically more favorable configurations with more nearest neighbors.

Using our destabilization technique, we thus have the unique opportunity to directly compare the rheological behavior of undisturbed particle gels of significantly different microstructures, produced by the $\Delta \mathrm{pH}$ and the $\Delta I$ method, respectively. In addition, we are able to compare the in situ properties to those obtained after altering the microstructure by shearing for both kinds of gels. Because the chemical composition and thus also the interparticle forces do not change during shearing, we obtain information on the influence of microstructure on the mechanical properties of concentrated particle networks.

\section{Experimental: Materials and Methods}

\section{In situ destabilization of concentrated colloids}

Direct coagulation casting (DCC; Gauckler et al., 1999a,b) is one of the few methods that allow a true in situ destabilization of charge-stabilized colloidal suspensions. It is based on timedelayed internal chemical reactions, such as enzyme-catalyzed hydrolysis reactions, or hydrolysis reactions induced by elevated temperatures. In this study, we use the hydrolysis of urea, catalyzed by the enzyme urease

$$
\mathrm{CO}\left(\mathrm{NH}_{2}\right)_{2}+2 \mathrm{H}_{2} \mathrm{O} \rightarrow \mathrm{NH}_{4}^{+}+\mathrm{NH}_{3}+\mathrm{HCO}_{3}^{-}
$$

where the onset and speed of the reaction is controlled by both temperature and enzyme concentration. The products of this reaction form a buffer system at $\mathrm{pH}$ 9. As the reaction proceeds, the suspension's $\mathrm{pH}$ is shifted toward the buffer $\mathrm{pH}$. At $\mathrm{pH} \mathrm{9,} \mathrm{the} \mathrm{ionic} \mathrm{strength} \mathrm{in} \mathrm{the} \mathrm{suspension} \mathrm{then} \mathrm{increases}$ homogeneously and continuously.

Because the isoelectric point (IEP) of alumina is found at around $\mathrm{pH} 9$ as well, the reaction of Eq. 1 can be used to destabilize alumina suspensions by shifting their $\mathrm{pH}$ from low values toward the IEP ( $\Delta \mathrm{pH}$ method). For particles with an IEP far from $\mathrm{pH} 9$ the urea-urease reaction leads to destabilization by an increase of ionic concentration at a constant $\mathrm{pH}$ of $9(\Delta I$ method). In the present study, the IEP of alumina particles used for destabilization by the $\Delta I$ method is changed by adding diammonium citrate as a surfactant (see below). We are thus 
able to destabilize alumina suspensions by either the $\Delta \mathrm{pH}$ method or the $\Delta I$ method using the urea-urease reaction in both cases.

\section{Materials}

For the suspensions we use high-purity $\alpha-\mathrm{Al}_{2} \mathrm{O}_{3}$ powder (Ceralox, HPA, 0.5 wt \% MgO, Condea Vista Co., Ceralox Division, Tucson, AZ) with an average particle diameter $\left(d_{50}\right)$ of $360 \mathrm{~nm}$, a specific surface area of $8 \mathrm{~m}^{2} / \mathrm{g}$, and a density of $3.98 \mathrm{~g} / \mathrm{cm}^{3}$. Zeta potential measurements, performed with an acoustic spectrometer (DT-1200, Dispersion Technology Inc., Bedford Hills, NY), yield an IEP at around $\mathrm{pH} 9$ for untreated alumina powders.

Other chemicals used in our experiments are hydrochloric acid (Merck, Darmstadt, Germany), diammonium citrate (Alfa Aesar, Johnson Matthey GmbH, Karlsruhe, Germany), urea (Sigma U-0631, Sigma-Aldrich Chemicals, Steinheim, Germany), urease (62,000 units/g, Roche Diagnostics $\mathrm{GmbH}$, Mannheim, Germany), and deionized water (resistivity $>18$ $\mathrm{M} \Omega \mathrm{cm}$ ). (One unit (U) of urease is defined as the amount needed to hydrolyze $1 \mu \mathrm{mol}$ of substrate per minute at $\mathrm{pH} 7.6$ and at a temperature of $25^{\circ} \mathrm{C}$.)

\section{Suspension preparation}

Gels are prepared at various particle volume fractions $\phi$, ranging between $\phi=25 \mathrm{vol} \%$ and $\phi=59 \mathrm{vol} \%$. Two different destabilization mechanisms are used, the $\Delta \mathrm{pH}$ and the $\Delta I$ methods.

Suspensions for $\Delta p H$ Destabilization. Deionized water is mixed with alumina powder and urea $(0.32 \mathrm{wt} \%)$ using a mechanical mixer. The $\mathrm{pH}$ of the suspension is then adjusted to $\mathrm{pH} \approx 4$ using hydrochloric acid ( $2 \mathrm{~mol} / \mathrm{L})$. Suspensions are subsequently dispersed by an ultrasonic treatment lasting 10 min [Ultrasonicator, UPS 200s (200 W), Dr. Hielscher GmbH, Teltow, Germany, cycle 0.5, amplitude 70\%]. The enzyme concentration is chosen as 1 unit per gram of alumina, which results in a shift of $\mathrm{pH}$ to the IEP within less than $2 \mathrm{~h}$. Before adding urease, the slurry is cooled to below $5^{\circ} \mathrm{C}$ in ice water. At this temperature, the enzyme-catalyzed hydrolysis reaction is very slow, which avoids any significant aggregation. Immediately after addition of the enzyme, the slurry is placed in the rheometer. As the temperature of the destabilized slurry increases, the speed of the enzymatic reaction is increased, which leads to an increase of $\mathrm{pH}$ and ultimately results in gelation. The samples are left undisturbed in the rheometer for $2.5 \mathrm{~h}$ before measurement.

Suspensions for $\Delta I$ Destabilization. For the case of ionic strength destabilization, higher amounts of urea are needed to achieve a destabilization of the system.

For the sample preparation, alumina powder, water, urea, and diammonium citrate (DAC) are mixed and then stirred for 15 min. DAC absorbs onto the surface of the particles and leads to a shift of the IEP of the alumina particles from $\mathrm{pH} 9$ to $\mathrm{pH} \approx$ 3.5 (Hidber and Gauckler, 1996). At low ionic strength, before the hydrolysis of urea is initiated, the suspension is thus electrostatically stabilized at a $\mathrm{pH}$ of 9 , the buffer $\mathrm{pH}$ of the products of Eq. 1. Relative to the weight of the alumina powder, we used 0.45 wt $\%$ of DAC in all our $\Delta I$ samples.

Dispersion is achieved by an ultrasonic treatment lasting 10 min (cycle: 0.5 , amplitude: $70 \%$ ). After addition of 75 units of urease per gram of alumina to the cooled slurry, the suspension is placed into the rheometer. Before measurement, samples are left undisturbed for around $16 \mathrm{~h}$.

\section{Rheological measurements}

Viscoelastic properties of the suspensions are investigated by rheological measurements using a Bohlin stress-controlled rheometer (Model CS-50, Bohlin Instruments, Studsvik, Sweden) equipped with a measuring tool of plate/plate geometry (serrated surface, 25-mm plate diameter). In determining yield strain and yield stress, there is always the fear that the data are contaminated with wall slip. We use serrated plates to prevent this problem (Walls et al., 2003). We have also performed test measurements with a vane tool geometry (Nguyen and Boger, 1983), which eliminates slip very efficiently. Results are comparable to those obtained in the parallel-plate geometry.

The temperature of our samples is controlled by a thermostat water bath and is kept constant at $25^{\circ} \mathrm{C}$. Evaporation of water is minimized by using a cylindrical cover above the sample (Bohlin Instruments). Oscillatory measurements are performed at a fixed frequency of $1 \mathrm{~Hz}$, varying the applied strain amplitude. From such an amplitude sweep curve, we evaluate the storage modulus $G^{\prime}$ in the linear viscoelastic regime, as well as the yield stress $\sigma_{y}$, formally defined as the minimum applied stress required to initiate rupture or flow in the system. In practice, the exact definition of the yield stress from a measured curve is more difficult and different methods have been proposed by various authors (Balzer et al., 1999b; Nguyen and Boger, 1983; Shih et al., 1999; Yanez et al., 1996). The yield stress is often defined as the critical stress where the phase angle reaches $45^{\circ}$ (that is, $G^{\prime}=G^{\prime \prime}$ ) in a stress ramp applied on an elastic network. Here, we use a method proposed by Yanez et al. (1996). They define the yield stress $\sigma_{y}$ as the product of the storage modulus $G^{\prime}$ in the linear viscoelastic regime and the yield strain $\gamma_{y}: \sigma_{y}=G^{\prime} \gamma_{y}$. The yield strain is determined as the intersection point of the regression line obtained in the linearly decreasing part of the storage modulus curve and the one obtained in the linear viscoelastic regime, as demonstrated in Figure 1. Our systems generally do not exhibit any significant frequency dependency of the storage modulus. We thus identify the storage modulus, as measured at a frequency of $1 \mathrm{~Hz}$ in the linear viscoelastic regime, as the plateau modulus $G_{p}^{\prime}$, which is usually determined by extrapolating $G^{\prime}(\omega)$ to zero frequency. Our amplitude sweep experiments are conducted with a gap of $1 \mathrm{~mm}$ between the parallel plates. Two different kinds of measurement are performed on a destabilized sample:

In Situ. In the in situ measurements, samples are placed directly inside the rheometer immediately after the addition of the enzyme urease. They are left undisturbed during the entire destabilization process. The rheological measurement is then directly started as a stress ramp, without applying any preshear.

In Situ, Presheared. Samples prepared and measured under in situ conditions are subjected to a second measurement. These samples have thus already been presheared, and their microstructure has been altered. To set reproducible starting conditions, we apply an additional preshear to the samples. In all measurements reported here, we apply a shear rate of $1 \mathrm{~s}^{-1}$ for $60 \mathrm{~s}$. Samples are then allowed to rest for an additional $60 \mathrm{~s}$. In measurements with different shear rates or resting times, we 


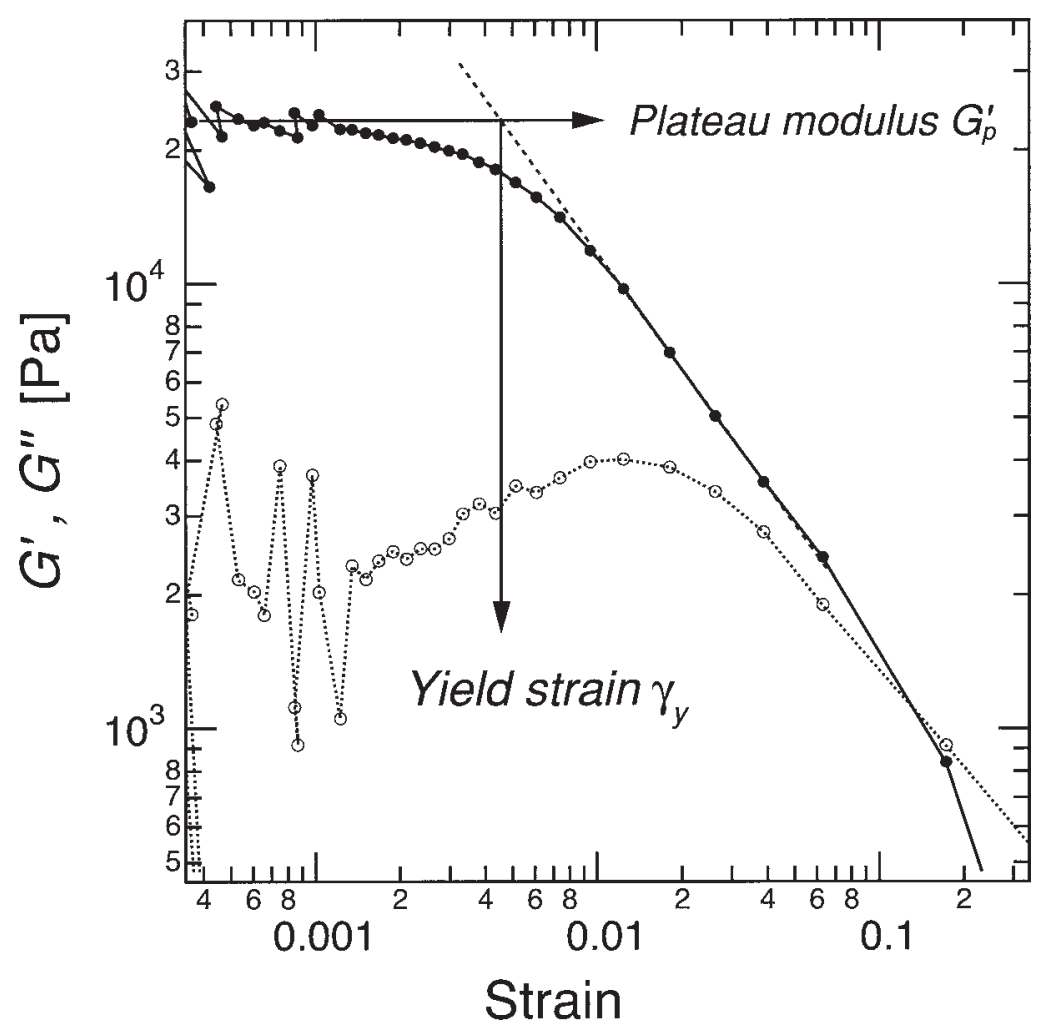

Figure 1. Method used for the determination of the plateau modulus and the yield strain, defined, as illustrated in the figure, by the intersection point of the regression line obtained in the linearly decreasing part of the storage modulus curve and the one obtained in the linear viscoelastic regime (after Wyss et al., 2002).

observe no significant changes in the resulting viscoelastic properties.

In addition to methods (i) and (ii), which are based on the hydrolysis of urea, we also produce gels ex situ by the conventional addition of base $(\mathrm{NaOH})$ or salt $\left(\mathrm{HH}_{4} \mathrm{Cl}\right)$.

\section{Results and Discussion}

\section{In situ measurements}

In Figure 2 we show the plateau modulus $G_{p}^{\prime}$ and the yield stress $\sigma_{y}$ for in situ destabilized $\Delta \mathrm{pH}$ and $\Delta I$ systems. We find that $\Delta I$ gels generally exhibit a significantly larger plateau modulus than those formed by the $\Delta \mathrm{pH}$ method. The dependency of the plateau modulus $G_{p}^{\prime}$ as a function of volume fraction $\phi$ can be approximated by a power-law $G_{p}^{\prime} \alpha \phi^{m}$ for both, the $\Delta \mathrm{pH}$ and the $\Delta I$ suspensions, as indicated by the fitted curves plotted in Figure 2a. Both systems show a comparable scaling behavior of the plateau modulus with very high powerlaw exponents $m$ of around 6. However, the scaling behavior of the yield strain is very different for the two systems. The yield strain of $\Delta I$ gels shows no dependency on volume fraction, whereas for $\Delta \mathrm{pH}$ gels it decreases strongly with increasing volume fraction, as shown in Figure $2 b$. This contrast in the yield behavior indicates a difference in the load-bearing mechanism of the two systems, as will be discussed below.

The inset of Figure 2 shows the yield stress for the two in situ systems as a function of volume fraction. We observe a crossover of the two curves at a volume fraction of around 30 vol $\%$. At lower volume fractions, the $\Delta I$ gels show a lower yield stress than that of $\Delta \mathrm{pH}$ gels, whereas for highly concentrated gels their yield stresses exceed those of $\Delta \mathrm{pH}$ gels. The transition occurs at a volume fraction of around $30 \mathrm{vol} \%$.

Highly concentrated $\Delta I$ gels thus show a higher plateau modulus and a higher yield stress compared to those of the corresponding $\Delta \mathrm{pH}$ gels. The same general behavior was observed in an earlier study on alumina wet green bodies (Balzer et al., 1999b), where the elastic and yield behavior in compression was measured at volume fractions above 50 vol $\%$.

\section{Scaling behavior}

We note that the scaling arguments given below should be handled with care for our highly concentrated gels. First, the range of volume fractions considered in this study cover merely half a decade. The accuracy of the exponents is therefore much lower than that for studies on diluted gels that can be studied over a very broad range of volume fractions. Second, the fractal description of network structures is generally believed to be no longer appropriate for highly concentrated gels. The size range where self-similar features can possibly be present is narrowed down dramatically with increasing volume fraction.

To account for the significant change in the yield strain dependency on volume fraction, we consider the scaling arguments introduced for fractal colloidal gels. Kantor and Webman (1984) showed that in the case of both central and noncentral interactions, the latter dominate for long particle chains They find the elastic constant $k_{e}$ of a particle chain to scale as 


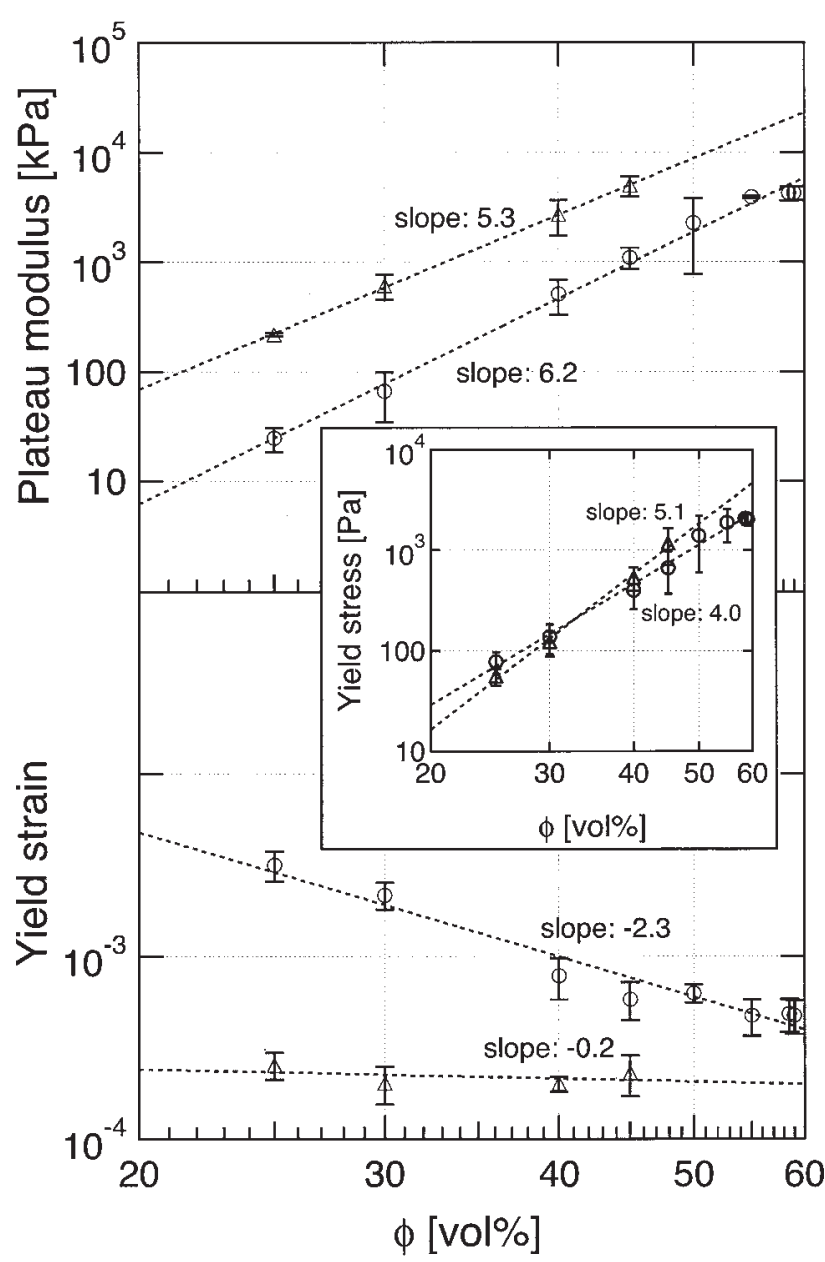

Figure 2. In situ measurements.

Comparison of elastic and yield behavior for particle gels formed by the $\Delta \mathrm{pH}$ (open symbols) and the $\Delta I$ method (solid symbols). (a) Plateau modulus vs. particle volume fraction. (b) Yield strain vs. particle volume fraction. Inset: Yield stress vs. particle volume fraction. The lines are power-law fits to the measured data.

$$
k_{e} \propto \frac{1}{N_{c h} R_{\perp}^{2}}
$$

where $R_{\perp}$ is the radius of gyration of the chain projected onto the plane perpendicular to the line connecting the ends of the chain and $N_{c h}$ is the number of particles in the chain. The parameter $R_{\perp}$ can be expressed as (de Rooij et al., 1994; Potanin, 1993)

$$
R_{\perp} \propto R_{c}^{\varepsilon}
$$

The parameter $\varepsilon$, with $0 \leq \varepsilon \leq 1$, then indicates the stressbearing mechanism of the chain. The case $\varepsilon=0$ applies to a straight chain, where the stress is transmitted by bond stretching. The case $\varepsilon=1$ describes a purely isotropic chain, where stress transmission through a bond-bending mechanism dominates.

Using Eq. 2, the external force $f_{\text {ext }}$ on a particle chain can be written as (de Rooij et al., 1994)

$$
f_{e x t}=k_{e} \Delta R_{c}=\frac{1}{R_{c}^{2 \varepsilon+d_{b}}} \frac{\Delta L}{L} R_{c}
$$

where the parameter $d_{b}$ is called the bond dimension or chemical dimension. It describes the scaling of the number of particles $N_{c h}$ in the chain with $R_{c}$ as $N_{c h} \propto R_{c}^{d_{b}}$.

We assume that a bond breaks if the force $f_{\text {ext }}$ on it exceeds a critical value. The yield strain will thus scale as

$$
\gamma_{y} \propto R_{c}^{2 \varepsilon+d_{b}-1}
$$

So far, a scaling behavior has only been assumed for the geometry of the particle chains within a network supporting the stress. Assuming a fractal structure, the size of aggregates $R_{c}$ scales as $R_{c} \propto \phi^{d_{f}-3}$, and thus the yield strain scales with volume fraction as

$$
\gamma_{y} \propto \phi^{\left(1-2 \varepsilon-d_{b}\right) /\left(3-d_{f}\right)}
$$

Even if a different scaling behavior of $R_{c}$ with volume fraction would apply for our concentrated structures, we can argue that the yield strain can be independent of the volume fraction only if $\varepsilon \approx 0$ and $d_{b} \approx 1$ (that is, for straight, rigid chains, where stresses are transmitted by a bond-stretching mechanism).

Accordingly, we attribute the stress-bearing mechanism of our $\Delta I$ gels to bond stretching, whereas for $\Delta \mathrm{pH}$ gels we attribute it to a bond-bending mechanism. To explain the very high exponents that we obtain for the scaling of the plateau modulus $G_{p}^{\prime}$ within a fractal scenario, we would have to assume unrealistically high values for the fractal dimension $d_{f}$.

\section{Measurements after disturbing the microstructure}

In the following, we compare the elastic and yield behavior of our gels before and after altering the microstructure by applying a large shear deformation.

$\Delta p H$ Gels. Curves for the $\Delta \mathrm{pH}$ gels are shown in Figure 3. After preshearing, our $\Delta \mathrm{pH}$ gels no longer show the strong dependency of the yield strain on the volume fraction, as shown in Figure $3 b$. Their yield strains are found at much lower values of around $2 \times 10^{-4}$, independent of volume fraction. This behavior indicates a fundamental change in the stressbearing mechanism of the gels. We suggest that the network changes from a homogeneous gel with a tortuous stress-bearing backbone to a less homogeneous gel, where stresses are transmitted through a bond-stretching mechanism. As a result of the stress-bearing mechanism changing from bond bending to bond stretching, we would then expect higher plateau moduli for the presheared gels. This is indeed what we find for our $\Delta \mathrm{pH}$ gels The plateau moduli of presheared $\Delta \mathrm{pH}$ gels are significantly higher than those for gels measured in situ, as shown in Figure 3a. In a fractal scenario, this would imply that the density of clusters increases and the homogeneity of the gel microstructure is reduced upon preshearing.

$\Delta I$ Gels. Curves for the $\Delta \mathrm{pH}$ gels are shown in Figure 4. The yield strain of presheared $\Delta I$ gels is lower than that for the corresponding in situ gels, as shown in Figure 4b. It shows a power-law dependency on volume fraction $\phi$ with an exponent of $m \approx-0.6$, indicating a less-rigid stress-bearing structure. 


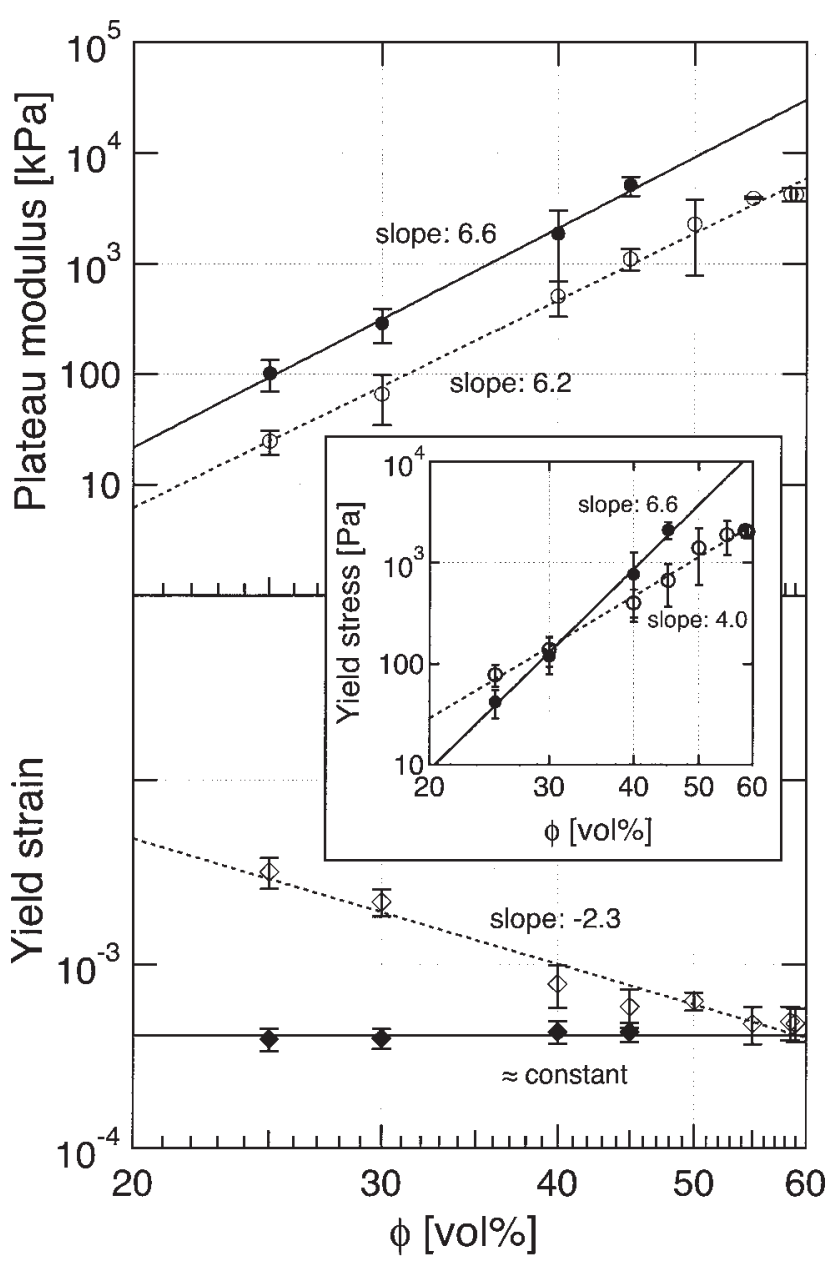

Figure 3. Elastic and yield behavior of the $\Delta \mathrm{pH}$ system measured in situ (open circles) and after preshearing (open triangles).

(a) Plateau modulus vs. particle volume fraction. (b) Yield strain vs. particle volume fraction. Inset: Yield stress vs. particle volume fraction. The lines are power-law fits to the measured data.

Accordingly, both the plateau moduli (Figure 4a) and the yield stresses (inset of Figure 4) are lower for the presheared gels than those for the in situ gels.

$\Delta p H$ Gels vs. $\Delta I$ Gels, Presheared. Finally, in Figure 5 we compare the properties of presheared gels produced by the $\Delta \mathrm{pH}$ and the $\Delta I$ methods, respectively. In significant contrast to the case of in situ gels, the presheared $\Delta \mathrm{pH}$ gels show both higher plateau modulus and higher yield stress than those of the corresponding $\Delta I$ gels at all explored volume fractions. The scaling behavior of both kinds of gels is in accord with a stress-bearing mechanism governed by bond stretching. Only a weak dependency of yield strain on volume fraction is observed for both $\Delta \mathrm{pH}$ and $\Delta I$ gels.

Suspensions destabilized ex situ by a conventional addition of acid/base or salt show an elastic and yield behavior comparable to that of presheared samples. Results for suspensions prepared by the addition of base $(\mathrm{NaOH})$ or salt $\left(\mathrm{NH}_{4} \mathrm{Cl}\right)$ are shown as single points in Figure 5.

Our results on the presheared gels are in good agreement with those obtained by Yanez et al. (1996) on alumina gels produced by the conventional addition of base or salt. They find a significantly higher plateau modulus and yield stress for gels formed at the isoelectric point (IEP) than for gels produced far from the IEP by the addition of salt. Various studies have shown both the yield stress and the storage modulus of presheared alumina gels to scale with volume fraction as a power law. The observed power-law exponents are typically in a range between 3 and 5 (Channell et al., 1997; Shih et al., 1999; Yanez et al., 1996), in good agreement with our measurements on presheared samples.

Presuming a comparable microstructure for the presheared samples, we attribute the differences in the magnitude of plateau modulus and yield stress to stronger interparticle forces for $\Delta \mathrm{pH}$-destabilized gels. Note that for the in situ samples, the magnitudes of plateau modulus and yield stress are reversed. Under the assumption that the preshearing procedure does not influence the interparticle forces, we conclude that the drastic changes in the viscoelastic behavior are caused by changes in microstructure and in the stress-bearing mechanisms of the gel networks.

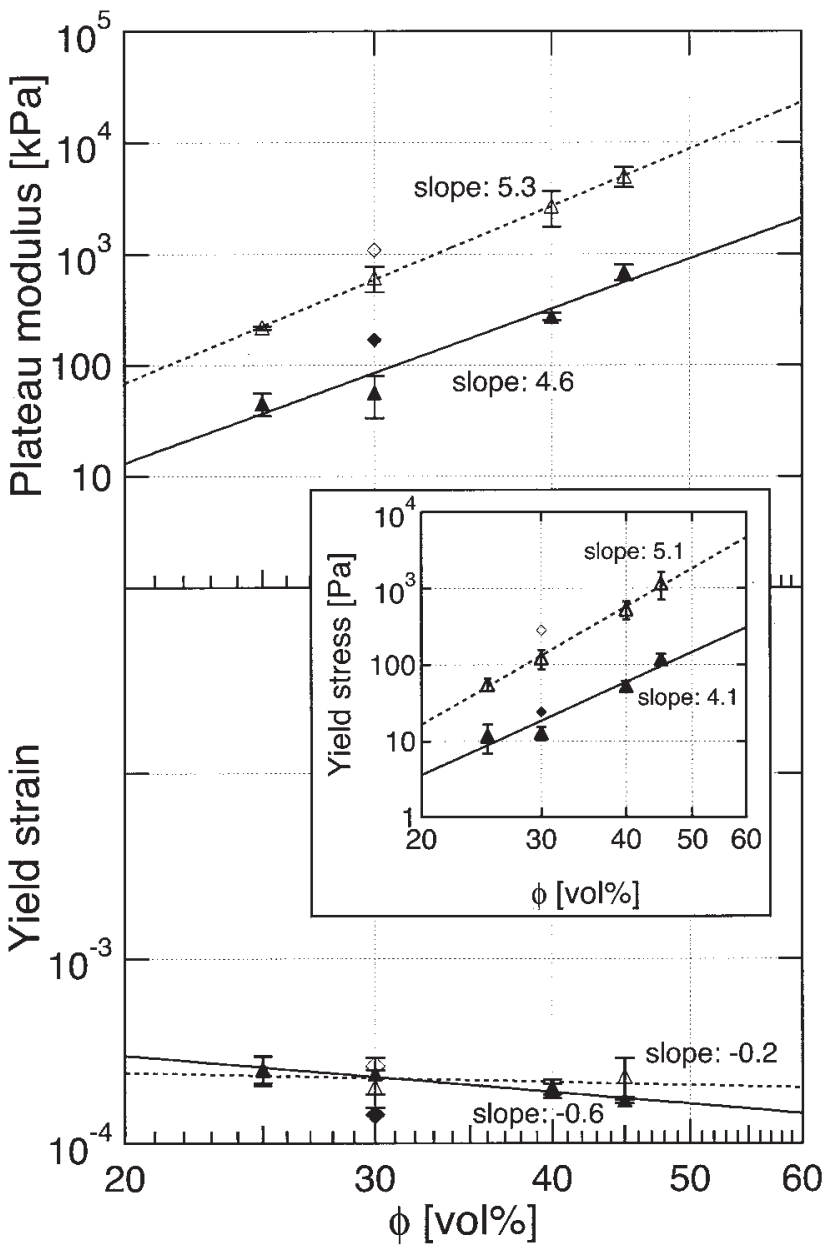

Figure 4. Elastic and yield behavior of the $\Delta /$ system measured in situ (solid circles) and after preshearing (solid triangles).

(a) Plateau modulus vs. particle volume fraction. (b) Yield strain vs. particle volume fraction. Inset: Yield stress vs. particle volume fraction. The lines are power-law fits to the measured data. 


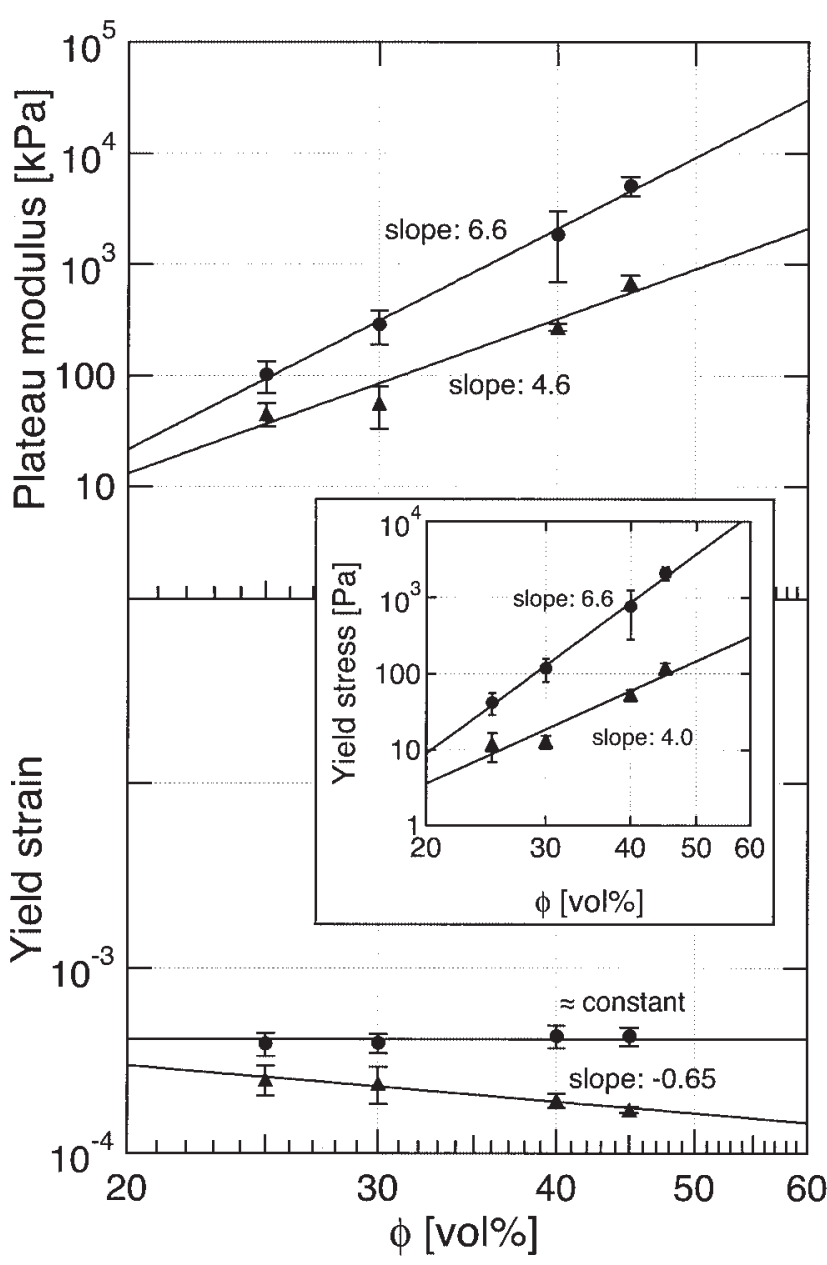

Figure 5. Measurements after disturbing the microstructure by preshearing.

Comparison of elastic and yield behavior for particle gels formed by the $\Delta \mathrm{pH}$ (open triangles) and the $\Delta I$ method (solid triangles). Results for gels obtained by the conventional addition of salt $\left(\mathrm{NH}_{4} \mathrm{Cl}\right)$ or base $(\mathrm{NaOH})$ are shown as solid diamonds or open diamonds, respectively. (a) Plateau modulus vs. particle volume fraction. (b) Yield strain vs. particle volume fraction. Inset: Yield stress vs. particle volume fraction. The lines are power-law fits to the measured data.

\section{Summary and Conclusions}

Our results illustrate the major influence of microstructure on the mechanical properties of particle gels. After in situ destabilization at high volume fractions, $\Delta I$ samples show a significantly higher plateau modulus compared to that of $\Delta \mathrm{pH}$ samples. However, after altering the microstructure of both kinds of gels by applying a large shear deformation, the elastic and yield properties change dramatically. We observe a strong increase of elastic properties for the $\Delta \mathrm{pH}$ gels. Moreover, after preshearing, the yield strain of $\Delta \mathrm{pH}$ gels shows only a very weak dependency on volume fraction, indicating a change of the stress-bearing mechanism from bond bending to bond stretching. For the $\Delta I$ destabilization, elastic and yield properties are decreased upon preshearing. The stress-bearing mechanism in the $\Delta I$ gels is attributed to bond stretching before and after applying a large shear deformation.

The microstructure of concentrated particle gels strongly influences their mechanical behavior. Our results suggest that the in situ $\Delta \mathrm{pH}$ destabilization is able to produce a homogeneous gel network, where the stress-bearing backbone consists of convoluted chains of single particles, which are able to support stresses predominantly by a bond-bending mechanism. Upon preshearing the $\Delta \mathrm{pH}$ gels, their stress-bearing mechanism is then irreversibly changed to bond stretching, which dramatically increases the elastic properties of the gels. $\Delta I$ gels respond to an external stress by bond stretching before and after altering their microstructures by shearing.

This interpretation is in agreement with results from previous studies (Wyss et al., 2001, 2002, 2004), indicating significant differences in microstructure for gels formed by the two different destabilization mechanisms. Combined with these findings, our results suggest a strong increase of elastic and yield properties of highly concentrated particle gels with decreasing homogeneity of their microstructures.

\section{Acknowledgments}

The authors thank Theo Tervoort and Véronique Trappe for fruitful discussions.

\section{Literature Cited}

Balzer, B., M. K. M. Hruschka, and L. J. Gauckler, "Coagulation Kinetics and Mechanical Behavior of Wet Alumina Green Bodies Produced via DCC," J. Colloid Interface Sci., 216(2), 379 (1999a).

Balzer, B., M. K. M. Hruschka, and L. J. Gauckler, "In-Situ Rheological Investigation of the Coagulation in Aqueous Alumina Suspensions," J. Am. Ceram. Soc., 84(8), 1733 (1999b).

Brown, W. D., and R. C. Ball, "Computer Simulation of Chemically Limited Aggregation," J. Phys. A, 18(9), L517 (1985).

Chang, J. C., F. F. Lange, and D. S. Pearson, "Viscosity and Yield Stress of Alumina Slurries Containing Large Concentrations of Electrolyte," J. Am. Ceram. Soc., 77(1), 19 (1994).

Channell, G. M., K. T. Miller, and C. F. Zukoski, "Effects of Microstructure on the Compressive Yield Stress," AICHE J., 46(1), 72 (2000).

Channell, G. M., and C. F. Zukoski, "Shear and Compressive Rheology of Aggregated Alumina Suspensions," AICHE J., 43(7), 1700 (1997).

de Rooij, R., D. van den Ende, M. H. G. Duits, and J. Mellema, "Elasticity of Weakly Aggregating Polystyrene Latex Dispersions," Phys. Rev. E, 49(4), 3038 (1994).

Fagan, M. E., and C. F. Zukoski, "The Rheology of Charge Stabilized Silica Suspensions," J. Rheol., 41(2), 373 (1997).

Gauckler, L. J., T. Graule, and F. Baader, "Ceramic Forming Using Enzyme Catalyzed Reactions," Mater. Chem. Phys., 2509, 1 (1999a).

Gauckler, L. J., T. J. Graule, F. Baader, and J. Will, "Enzyme Catalysis of Alumina Forming," Key Eng. Mater., 159-160, 135 (1999b).

Hidber, P. C., and L. J. Gauckler, "Citric Acid-A Dispersant for Aqueous Alumina Suspensions," J. Am. Ceram. Soc., 79(7), 1857 (1996).

Kantor, Y., and I. Webman, "Elastic Properties of Random Percolating Systems," Phys. Rev.Lett., 52(21), 1891 (1984).

Nguyen, Q. D., and D. V. Boger, "Yield Stress Measurements for Concentrated Suspensions," J. Rheol., 27(4), 321 (1983).

Potanin, A. A., "On the Computer Simulation of the Deformation and Breakup of Colloidal Aggregates in Shear Flow," J. Colloid Interface Sci., 157, 399 (1993).

Rueb, C. J., and C. F. Zukoski, "Viscoelastic Properties of Colloidal Gels," J. Rheol., 41(2), 197 (1997).

Shih, W. H., W. Y. Shih, S. I. Kim, J. Liu, and I. A. Aksay, "Scaling Behavior of the Elastic Properties of Colloidal Gels," Phys. Rev. A, 42(8), 4772 (1990).

Shih, W. Y., W.-H. Shih, and I. A. Aksay, "Elastic and Yield Behavior of Strongly Flocculated Colloids," J. Am. Ceram. Soc., 82(3), 616 (1999).

Walls, H. J., S. B. Caines, A. M. Sanchez, and S. A. Khan, "Yield Stress and Wall Slip Phenomena in Colloidal Silica Gels," J. Rheol., 47(4), 847 (2003).

Weitz, D. A., and M. Oliveria, "Fractal Structures Formed by Kinetic 
Aggregation of Aqueous Gold Colloids," Phys. Rev. Lett., 52(16), 1433 (1984).

Wu, H., and M. Morbidelli, "A Model Relating Structure of Colloidal Gels to Their Elastic Properties," Langmuir, 17, 1030 (2001).

Wyss, H. M., M. Hütter, M. Müller, L. P. Meier, and L. J. Gauckler, "Quantification of Microstructures in Stable and Gelated Suspensions from Cryo-SEM," J. Colloid Interface Sci., 248, 340 (2002).

Wyss, H. M., S. Romer, F. Scheffold, P. Schurtenberger, and L. J. Gauckler, "Diffusing-Wave Spectroscopy of Concentrated Alumina Suspensions during Gelation," J. Colloid Interface Sci., 241, 89 (2001).
Wyss, H. M., E. Tervoort, L. P. Meier, M. Müller, and L. J. Gauckler, "Relation between Microstructure and Mechanical Behavior of Concentrated Silica Gels," J. Colloid Interface Sci., 273(2), 455 (2004).

Yanez, J. A., T. Shikata, F. F. Lange, and D. S. Pearson, "Shear Modulus and Yield Stress Measurements of Attractive Alumina Particle Networks in Aqueous Slurries," J. Am. Ceram. Soc., 79(11), 2917 (1996).

Manuscript received Jul. 7, 2003, and revision received May 14, 2004. 